\title{
Prueba de caminata en seis minutos en sujetos chilenos sanos de 20 a 80 años
}

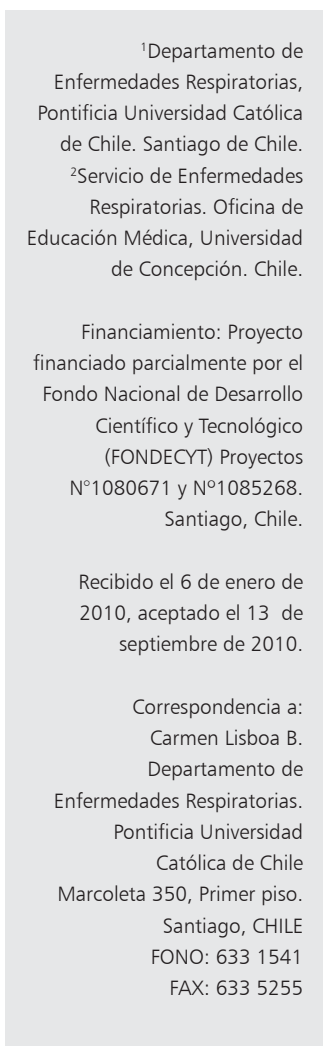

\author{
RODRIGO OSSES A. ${ }^{1}$, JORGE YÁÑEZ V. ${ }^{1}$, \\ PAULINA BARRÍA P. ${ }^{1}$, SYLVIA PALACIOS M. ${ }^{2}$, JORGE DREYSE D. ${ }^{1}$, \\ ORLANDO DÍAZ P. ${ }^{1}$, CARMEN LISBOA B. ${ }^{1}$
}

\section{Reference values for the 6-minutes walking test in healthy subjects $20-80$ years old}

\begin{abstract}
Background: The six minute walking distance test (6MWD) is widely used to evaluate exercise capacity in several diseases due to its simplicity and low cost. Aim: To establish reference values for $6 \mathrm{MWD}$ in healthy Chilean individuals. Material and methods: We studied 175 healthy volunteers aged 20-80 years (98 women) with normal spirometry and without history of respiratory, cardiovascular or other diseases that could impair walking capacity. The test was performed twice with an interval of $30 \mathrm{~min}$. Heart rate, arterial oxygen saturation (with a pulse oxymeter) and dyspnea were measured before and after the test. Results: Walking distance was $576 \pm 87 \mathrm{~m}$ in women and $644 \pm 84 \mathrm{~m}$ in men $(p<0.0001)$. For each sex, a model including age, height and weight produced $6 M W D$ prediction equations with a coefficient of determination $\left(R^{2}\right)$ of 0.63 for women and 0.55 for men. Conclusions: Our results provide reference equations for $6 M W D$ that are valid for healthy subjects between 20 and 80 years old.
\end{abstract}

(Rev Med Chile 2010; 138: 1124-1130).

Key words: Functional residual capacity; Health status; 6-minutes walking test.

L

a caminata en seis minutos es una prueba de ejercicio modificada de la realizada en

12 minutos, descrita en 1976 por McGavin y cols ${ }^{1,2}$. En los últimos años ha adquirido una importancia progresiva por su fácil implementación y reproducibilidad. Se ha utilizado ampliamente en pacientes con enfermedades respiratorias y cardiovasculares; para evaluar la capacidad de ejercicio de los pacientes ${ }^{3-8}$; la respuesta a intervenciones terapeúticas ${ }^{9,10}$; como factor pronóstico de sobrevida ${ }^{6,7,11,12} ;$ y como índice de deterioro en la enfermedad pulmonar obstructiva crónica $(\text { EPOC) })^{13,14}$.

Dentro de sus ventajas destaca el no requerir equipos de alto costo, como tampoco de un entrenamiento exhaustivo para quien lo ejecuta. Prácticamente carece de riesgos para el paciente, refleja en forma adecuada la capacidad funcional del individuo en sus actividades diarias y se ha determinado el cambio mínimo clínicamente relevante $^{5,15,16}$.

Para su correcta interpretación se requiere de valores de referencia de la población a la cual se le efectúa esta prueba ${ }^{17-21}$. En Chile no se dispone de estos valores de referencia, por lo que se utilizan ecuaciones obtenidas en estudios extranjeros ${ }^{17,18}$, que pudieran no reflejar nuestra realidad.

El propósito de este estudio ha sido obtener valores normales de la prueba de caminata $6 \mathrm{mi}-$ nutos en población chilena adulta sana y generar ecuaciones de regresión que permitan establecer valores de referencia para su uso en los laboratorios de función pulmonar en nuestro país.

Los objetivos secundarios fueron comparar 
la ecuación con las disponibles en la literatura, evaluar el efecto del aprendizaje en dos pruebas realizadas con una diferencia de 30 minutos y establecer la respuesta de la frecuencia cardiaca, de la magnitud de los cambios en la disnea y de la saturación de oxígeno al final de la prueba.

\section{Métodos}

Sujetos

Se estudiaron 175 sujetos (98 mujeres), voluntarios sanos, con edades entre 20 y 80 años, habitantes de las ciudades de Santiago y Concepción, que aceptaron participar en el protocolo firmando un acta de consentimiento informado aprobada por los Comités de Ética de ambas instituciones. Todos los participantes fueron reclutados entre el personal de ambas instituciones y sus familiares. Se consideraron criterios de exclusión los antecedentes de asma u otra enfermedad respiratoria, síndrome coronario reciente, arritmias, hipertensión arterial no controlada, insuficiencia respiratoria y enfermedades neuromusculares que impidieran la adecuada realización del examen. Se aplicó la escala de Charlson ${ }^{22}$ para evaluar comorbilidades asociadas.

\section{Mediciones}

En todos los sujetos se midió el peso, la talla y se calculó el índice de masa corporal (IMC).

La espirometría se efectuó siguiendo las recomendaciones propuestas por las Sociedades Americana de Tórax (ATS) y Europea de Enfermedades Respiratorias (ERS) ${ }^{23}$.

La prueba de caminata en seis minutos se realizó en dos oportunidades en un mismo día, separada por 30 minutos, en un corredor de 30 metros de largo ubicado en el interior de los respectivos laboratorios, de acuerdo a las recomendaciones de la $\mathrm{ATS}^{15}$. Los sujetos fueron estimulados cada minuto con las frases sugeridas por las normas de la ATS, con el propósito de que caminaran lo más posible en los 6 minutos. Al inicio y al final de la prueba, se midió frecuencia cardiaca y saturación arterial de oxígeno $\left(\mathrm{SpO}_{2}\right)$ con un oxímetro de pulso (Nonin Medical Inc., Plymouth, Mn., USA) y el grado de dificultad respiratoria mediante la escala psicofísica de Borg ${ }^{24}$.

\section{Estadística}

Los resultados del texto, tablas y figuras se expresan como promedios \pm 1 DE. Las compa- raciones entre ambos sexos se realizaron con la prueba de $t$ de Student para muestras independientes. Los cambios de la frecuencia cardiaca, $\mathrm{SpO}_{2}$ y disnea entre el inicio y el final de la mejor caminata se analizaron con la prueba de t para muestras pareadas. Se empleó correlación simple de Pearson y análisis de regresión múltiple para evaluar las variables independientes que explican la varianza de la DR6.

\section{Resultados}

Características generales. Las características del grupo de sujetos estudiados, separados por sexo, se comparan en la Tabla 1. Se observaron diferencias significativas en la talla y en el peso, siendo el IMC semejante en ambos. Los valores espirométricos estuvieron dentro de límites normales para edad, talla y sexo.

Distancia recorrida. En el grupo total, la DR6 fue de $588 \pm 93 \mathrm{~m}$ en la primera prueba y de $602 \pm$ $92 \mathrm{~m}$ en la segunda, representando un incremento de $13 \pm 24 \mathrm{~m}(2,5 \pm 5 \%)$. Individualmente, este aumento de la DR6 en la segunda prueba se observó en 132 sujetos $(75,4 \%)$, mientras que en 41 $(23,4 \%)$ disminuyó, y en 2 no cambió.

La mejor DR6 registrada para cada sujeto fue significativamente superior en hombres que en mujeres (644 \pm 84 vs $576 \pm 87 \mathrm{~m}$; p < 0,0001). La Figura 1 muestra la DR6 en las distintas categorías de edad entre los 20 y 80 años, en ambos sexos. Se observó una disminución sostenida a medida que aumenta la edad, a la vez que una diferencia a favor

Tabla 1. Características generales de los 175 sujetos estudiados

\begin{tabular}{|lcrc|}
\hline & $\begin{array}{c}\text { Mujeres } \\
\mathbf{n}=\mathbf{9 8}\end{array}$ & $\begin{array}{c}\text { Hombres } \\
\mathbf{n}=\mathbf{7 7}\end{array}$ & $\mathbf{p}$ \\
\hline Edad, años & $56 \pm 16$ & $52 \pm 17$ & 0,093 \\
\hline Talla, cm & $158 \pm 6$ & $171 \pm 7$ & $<0,0001$ \\
\hline Peso, $\mathrm{kg}$ & $64 \pm 9$ & $78 \pm 11$ & $<0,0001$ \\
\hline IMC, $\mathrm{kg} \cdot \mathrm{m}^{2}$ & $26 \pm 4$ & $27 \pm 3,5$ & 0,064 \\
\hline DR6, metros & $576 \pm 87$ & $644 \pm 84$ & $<0,0001$ \\
\hline
\end{tabular}

IMC: índice de masa corporal; DR6: distancia recorrida en la caminata en 6 minutos. 


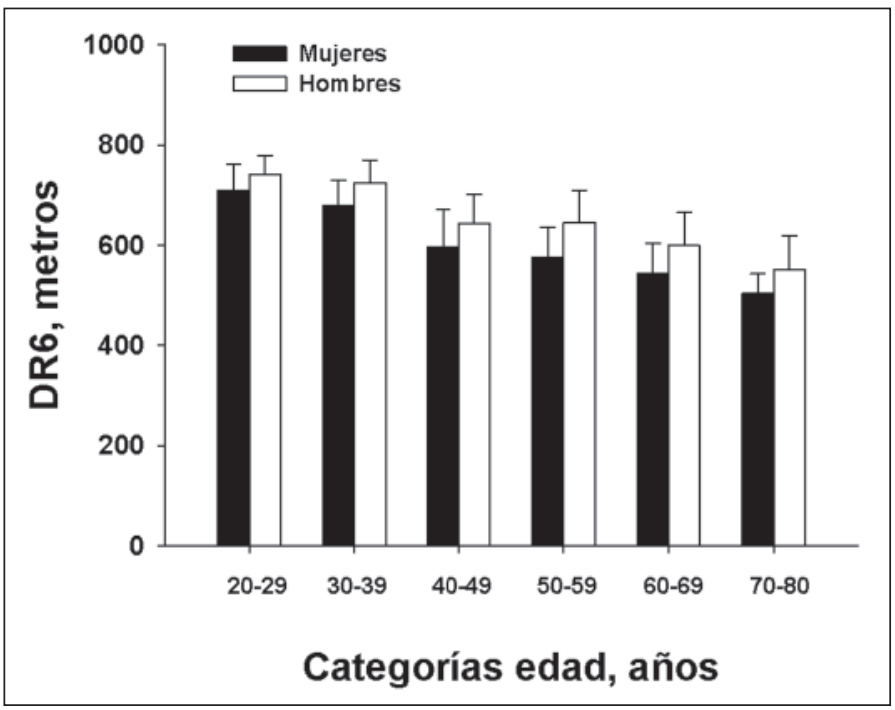

Figura 1. Distancia recorrida durante la caminata en 6 minutos (DR6) en las distintas categorías de edad y según sexo.

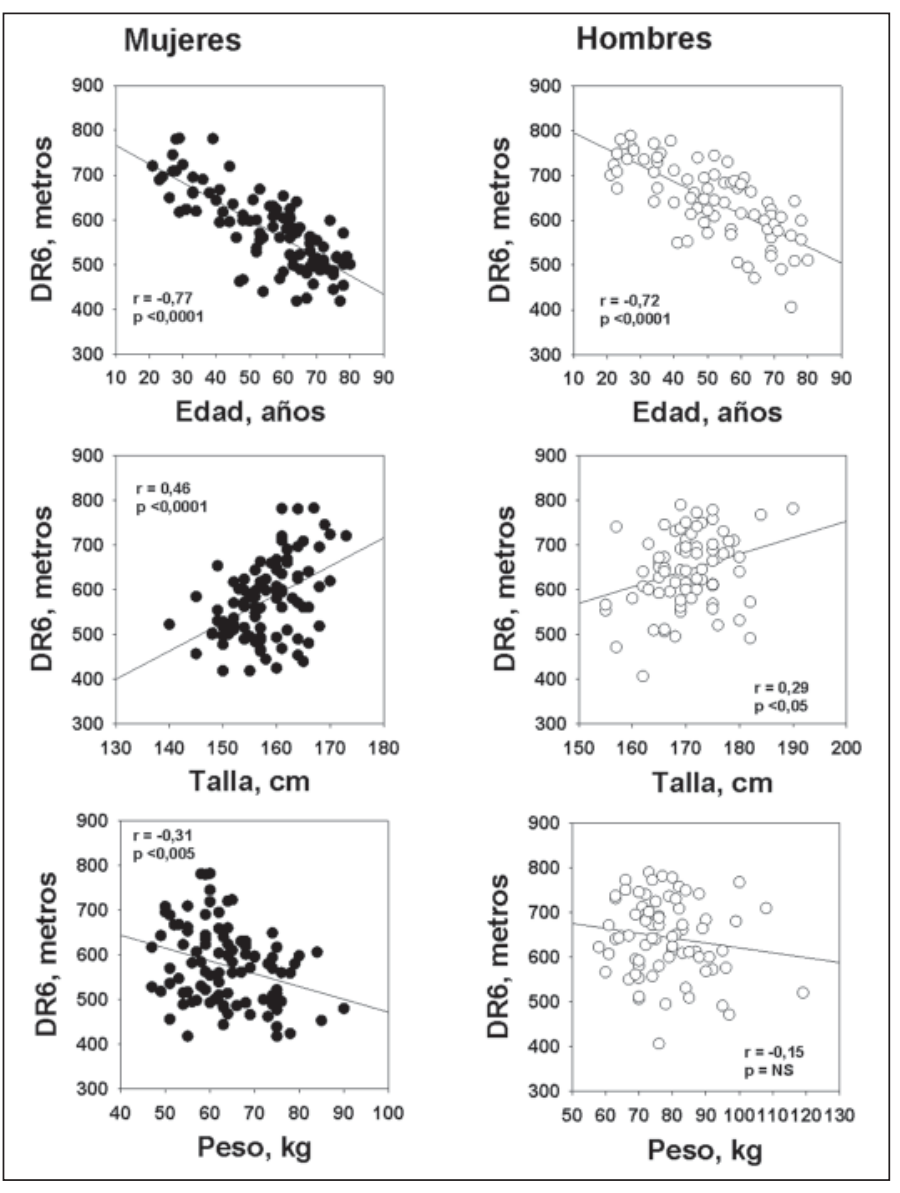

de los hombres para cada rango, similar a la descrita para el grupo total.

La Figura 2 y la Tabla 2 muestran las correlaciones entre la edad, las características antropométricas de los sujetos, y la DR6. En el grupo total, se observaron correlaciones significativas entre edad, talla e IMC con DR6. Al separar el análisis por sexos, la edad, talla, peso e IMC se correlacionaron significativamente con la DR6 en las mujeres, mientras que en los hombres esto sólo ocurrió con la edad, talla e IMC.

Ecuaciones de predicción. Con los valores obtenidos se generaron ecuaciones de regresión para ambos sexos, las que se describen en la Tabla 3.

En la Figura 3 se comparan mediante gráficos de identidad los valores de referencia obtenidos para cada uno de nuestros sujetos con las ecuaciones de Troosters y cols ${ }^{18} \mathrm{y}$ de Enright y $\mathrm{cols}^{17}$. Se observa que la primera subvalora nuestros resultados y la segunda los sobrevalora.

Cambios de los indices medidos al final de la prueba. Los cambios experimentados entre la situación basal y el final de la caminata para la frecuencia cardiaca, $\mathrm{SpO}_{2}$ y disnea se describen en la Tabla 4. $\mathrm{La} \mathrm{SpO}_{2}$ disminuyó en 37 sujetos $(-1,3 \pm 0,8 \%$; rango: -1 a -4$)$, mientras que en 138 aumentó o no experimentó cambios. La FC aumentó en todos los sujetos (+55 \pm 19 latidos/min; rango: 12110), siendo el aumento similar en ambos sexos.

Figura 2. Correlaciones entre la distancia recorrida durante la caminata en 6 minutos (DR6), edad, talla y peso en mujeres y hombres. 


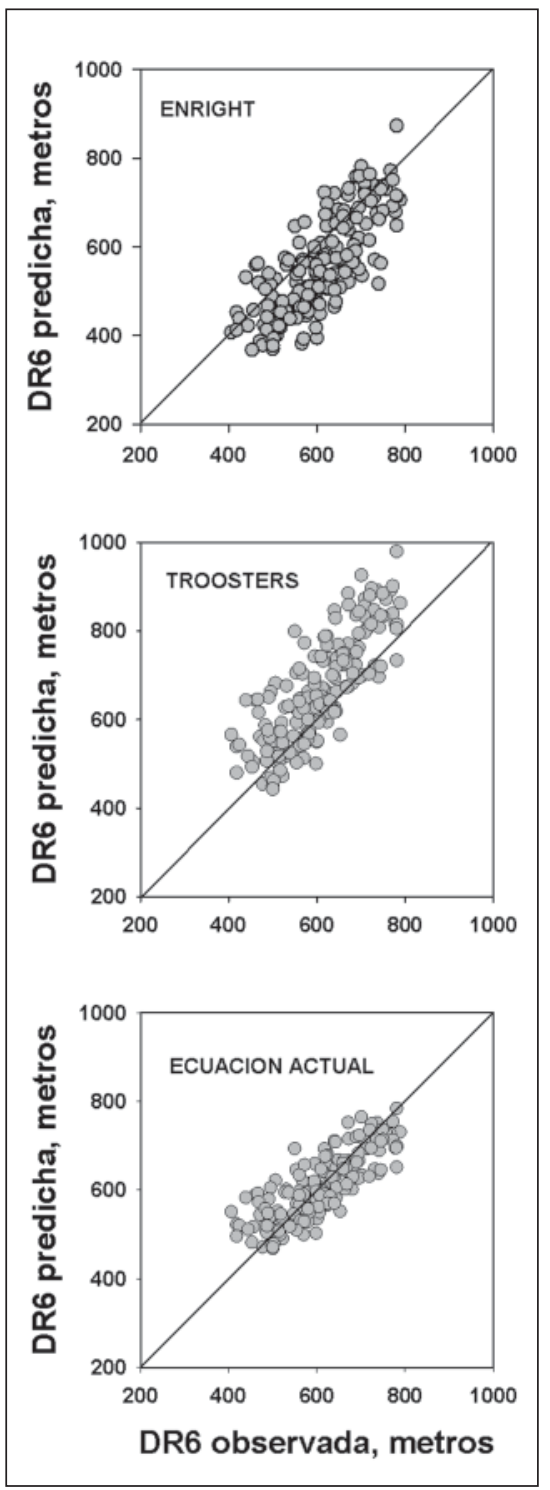

Tabla 2. Correlaciones entre la distancia recorrida durante la caminata en 6 minutos en el grupo total y en ambos sexos

\begin{tabular}{|c|c|c|c|}
\hline & \multicolumn{3}{|c|}{ DR6, metros } \\
\hline & Total & Mujeres & Hombres \\
\hline Edad, años & $-0,74^{\ddagger}$ & $-0,77^{\ddagger}$ & $-0,72^{\ddagger}$ \\
\hline Talla, cm & $0,51^{\ddagger}$ & $0,46^{\ddagger}$ & $0,29^{*}$ \\
\hline Peso, kg & 0,04 & $-0,31^{\dagger}$ & $-0,15$ \\
\hline $\mathrm{IMC}, \mathrm{kg} \cdot \mathrm{m}^{2}$ & $-0,34^{\ddagger}$ & $-0,50^{\ddagger}$ & $-0,32^{+}$ \\
\hline
\end{tabular}

DR6: distancia recorrida en la caminata en 6 minutos; IMC: índice de masa corporal. ${ }^{*} p<0,05 ;{ }^{\dagger} p<0,005 ;{ }^{\ddagger} p<0,0001$.

Tabla 3. Ecuaciones de regresión para la caminata en 6 minutos en hombres y mujeres

\section{Mujeres}

DR6, metros $=457-3,46 \times$ Edad (años) $+2,61 \times$ Talla $(\mathrm{cm})-1,57 \times$ Peso $(\mathrm{kg}) \pm 53$ (EE)

$R^{2}=0,63$

\section{Hombres}

DR6, metros $=530-3,31 \times$ Edad (años) $+2,36 \times$ Talla $(\mathrm{cm})-1,49 \times$ Peso $(\mathrm{kg}) \pm 58(\mathrm{EE})$

$R^{2}=0,55$

DR6: distancia recorrida en la caminata en 6 minutos; EE: error estándar de la estimación.

Figura 3. Gráficos de identidad que comparan los valores obtenidos en el presente estudio para la distancia recorrida en la caminata en 6 minutos (DR6) con los valores predichos por diferentes ecuaciones.

Tabla 4. Saturación de oxígeno, frecuencia cardiaca y disnea al inicio y final de la caminata en 6 minutos

\begin{tabular}{|lllllll|}
\hline & \multicolumn{3}{c}{ Mujeres } & \multicolumn{3}{c|}{ Hombres } \\
& Basal & Final & D & Basal & Final & D \\
\hline $\mathrm{SpO}_{2^{\prime}} \%$ & $96,8 \pm 1,5$ & $97,5 \pm 1,7$ & $0,7 \pm 1,4$ & $97 \pm 1,3$ & $97 \pm 1,6$ & $0,0 \pm 1,4$ \\
$\begin{array}{l}\mathrm{FC} \text { l latidos } / \\
\text { min }\end{array}$ & $74 \pm 10$ & $129 \pm 26$ & $54 \pm 23$ & $75 \pm 9$ & $128 \pm 23$ & $54 \pm 22$ \\
\hline Disnea, Borg & $0,0 \pm 0,0$ & $2,2 \pm 1,5$ & $2,2 \pm 1,5$ & $0 \pm 0$ & $2,3 \pm 1,5$ & $2,3 \pm 1,5$ \\
\hline
\end{tabular}

$\mathrm{SpO}_{2}$ : saturación arterial de oxígeno medida con oxímetro de pulso; FC: frecuencia cardíaca. 


\section{Discusión}

El presente estudio proporciona ecuaciones para predecir la distancia recorrida en 6 minutos en la prueba de caminata, para hombres y mujeres entre 20 y 80 años obtenidos en forma prospectiva en una población de voluntarios sanos residentes de Santiago y Concepción. Los modelos incluyeron el género, la edad, la talla y el peso, siendo los coeficientes de determinación $\mathrm{R}^{2}$ de 0,63 y 0,55 para mujeres y hombres, respectivamente, confirmando que estos factores pueden predecir en forma confiable la C6M. Nuestros datos proporcionan además información sobre los cambios de $\mathrm{SpO}_{2}$, frecuencia cardiaca y disnea que se producen entre el reposo en posición de pies antes de iniciar la prueba e inmediatamente de finalizada ésta, índices que son frecuentemente medidos y sobre los cuales existe escasa información.

Los resultados confirman que la distancia recorrida en seis minutos es mayor en los hombres, disminuye con la edad y tiene una relación directamente proporcional con la talla e inversa con el peso.

La falta de valores de referencia de la caminata obtenidos en nuestra población ha determinado que se utilicen valores teóricos foráneos. Los más utilizados son los propuestos por Enright y Sherril ${ }^{17}$ obtenidos en 253 mujeres y 205 hombres entre 40 y 80 años de población americana. Estos autores realizaron una sola prueba y los sujetos fueron instruidos para caminar a su propio paso sin ser estimulados durante la prueba. Es muy probable que debido a estas dos diferencias metodológicas, los valores del presente estudio expresados en porcentaje de dichos valores teóricos aparezcan sobrevalorados. Esto se explicaría porque se ha comunicado hasta $30 \%$ de diferencia en la C6M si el sujeto es o no es estimulado. Los otros valores de referencia utilizados con frecuencia son los de Troosters y $\operatorname{cols}^{18}$ realizados en Bélgica, quienes estudiaron una muestra menor de sujetos, compuesta por 72 sujetos de ambos géneros, con edades entre 50 y 85 años. Los sujetos realizaron la prueba en 2 oportunidades, separadas aproximadamente por 2,5 horas y fueron estimulados cada 30 segundos para caminar lo más rápidamente posible. La segunda prueba fue $8 \pm 5 \%$ mayor que la primera. Los autores observaron una importante variabilidad en la prueba que tuvo un rango entre 383 y 820 m con un promedio de $631 \pm 93$ metros, siendo 84 metros mayor en los hombres. Su ecuación subvalora en forma importante a los sujetos del presente estudio.

En la literatura que revisamos encontramos sólo dos estudios realizados en individuos más jóvenes. En uno de ellos, llevado a cabo por Gibbons y cols ${ }^{19}$, los sujetos reclutados tenían entre 20 y 80 años, mientras que en el otro, efectuado por Chetta y cols ${ }^{21}$, tenían entre 20 y 50 años. Nuestro estudio, que también incluye sujetos con un amplio rango de edad, puede ser útil para evaluar la capacidad de ejercicio en pacientes con diversas enfermedades que se presentan en grupos etarios muy diferentes, como es el caso de la fibrosis quística y la EPOC, entre otras.

Las diferencias observadas según la ecuación utilizada, ponen de manifiesto la importancia de contar con valores nacionales para expresar las pruebas funcionales respiratorias, teniendo en consideración que los valores de referencia de esta prueba pueden depender, además, de las características demográficas, de la raza, del estilo de vida y del grado de actividad física diaria de los individuos. En el análisis del estudio multinacional de Celli y cols..$^{25}$ que describe en pacientes con EPOC el índice de gravedad BODE, que incluye la prueba de caminata en 6 minutos, y que fue realizado en residentes de USA, España y Venezuela es llamativo el hecho de que los pacientes españoles tienen, para un mismo grado de obstrucción bronquial, una C6M (metros) muy superior a la encontrada en los pacientes provenientes de Estados Unidos. Una comunicación preliminar reciente de Casanova y cols. ${ }^{26}$ de un estudio multicéntrico realizado en sujetos normales provenientes de España y Sudamérica, la C6M fue más alta en estos últimos ${ }^{26}$.

Por tratarse de una prueba de ejercicio submáxima, que se utiliza frecuentemente en clínica, la información sobre la respuesta de la frecuencia cardiaca, los cambios de la $\mathrm{SpO}_{2}$ y de la disnea en sujetos normales resulta de gran interés, especialmente en los sujetos de mayor edad. Enright y Sherril ${ }^{17}$ comunicaron un aumento promedio de 20 latidos/min, con rango entre $-6 \mathrm{y}+58$, en las mujeres y de 25 latidos/min en los hombres, con un rango entre $-8 \mathrm{y}+79$. Chetta $\mathrm{y} \mathrm{col}^{21}$ observaron que la frecuencia cardiaca aumentó en las mujeres desde $81 \pm 10$ a $132 \pm 18$ latidos/min y en los hombres de $75 \pm 10$ a $119 \pm 18$ latidos/min, lo que representó en el grupo total $67 \pm 10 \%$ de la frecuencia cardiaca máxima. En el presente estudio 
se observó un aumento desde $74 \pm 10$ a $129 \pm 22$ en las mujeres y desde $75 \pm 11$ a $130 \pm 21$ latidos/min en los hombres. Tal frecuencia cardíaca del final de la prueba corresponde a $74 \pm 12 \%$ y $74 \pm 11 \%$ de la frecuencia cardiaca máxima, respectivamente, calculada como $210-0,66$ x edad. ${ }^{27}$

En el estudio de Enright y Sherril ${ }^{19}$ la $\mathrm{SpO}_{2}$ como promedio no cambió, pero los rangos fueron de $-16 \mathrm{a}+2 \%$ en las mujeres y de $-8 \mathrm{a}+2 \%$ en los hombres. Chetta y cols ${ }^{21}$ tampoco encontraron cambios significativos en la $\mathrm{SpO}_{2}$ con el ejercicio, observando un leve descenso de $1 \pm 1 \%$. Nuestros resultados confirman los hallazgos de los estudios anteriores, al no demostrar cambios de la $\mathrm{SpO}_{2}$ entre el reposo y el final de la C6M. Dos sujetos presentaron una caída de $4 \%$ en relación al valor basal y otro una reducción de $3 \%$, situación que puede ocurrir en sujetos normales durante un ejercicio máximo y que puede considerarse como desaturación leve, pues está en el rango 3-4\% inferior a la basal ${ }^{27}$.

Nuestro estudio tiene algunas limitaciones. Las ecuaciones no se obtuvieron de una muestra poblacional, sino de voluntarios. El estudio fue efectuado en dos centros distintos y por diferentes ejecutores de la prueba. Sin embargo, el protocolo se ajustó a las recomendaciones de la ATS, por lo que nos parece que este punto no es relevante. Tampoco se trata de una población completamente sana, pues en los sujetos de mayor edad varios presentaban comorbilidades, mayoritariamente hipertensión arterial controlada en tratamiento (12 sujetos) y diabetes mellitus estable ( 5 sujetos). Sin embargo, la presión arterial en reposo era normal, sus valores espirométricos estaban dentro de límites normales y la $\mathrm{SpO}_{2}$ en reposo era normal y no descendió con el ejercicio. Ninguno de los sujetos se detuvo durante la prueba y la respuesta cardiaca correspondió aproximadamente al 74\% de la máxima. Consideramos, por lo tanto, que a pesar de la presencia de comorbilidades en los sujetos mayores, la muestra es representativa de la población.

En conclusión, hemos establecido valores de referencia de la caminata en seis minutos en una población chilena mayoritariamente sana, generando una ecuación de regresión que puede considerarse para su empleo en los laboratorios de función pulmonar de nuestro país debido a las diferencias detectadas con las otras ecuaciones en uso actualmente.

\section{Referencias}

1. Butland RJA, Pamg J, Gross ER, Woodcock AA, Geddes DM. Two, six and twelve-minute walking tests in respiratory diseases. BMJ 1982; 284: 1607-8.

2. McGavin CR, Gupta SP, McHardy GRJ. Twelve minute walking test for assessing disability in chronic bronchitis. BMJ 1976; 1: 822-3.

3. Casas A, Vilaro J, Rabinovich R, Mayer AM, Barberá JA, Rodriguez-Roisin R, et al. Encouraged 6-min walking test indicates maximum sustainable exercise in COPD patients. Chest 2005; 128: 55-61.

4. Rabinovich RA, Vilano J, Roca J. Evaluación de la tolerancia al ejercicio en pacientes con EPOC. Prueba de marcha en 6 minutos. Arch Bronconeumol 2004; 40: 80-5.

5. Carter R, Holiday P, Nwasuruba C, Stocks J, Grothues C. Six-minute walk work for assessment of functional capacity in patients with COPD. Chest 2003; 123: 1408-15.

6. Lederer D, Arcasoy S, Wilt J, D’Ovidio F, Sonett J, Kawut S. Six-minute-walk distance predicts waiting list survival in idiopathic pulmonary fibrosis. Am J Respir Crit Care Med 2006; 174: 659-64.

7. Shah M, Hasselblad V, Gheorghiade M, Adams K, Swedberg K, Califf R, et al. Prognostic usefulness of the six-minute walk test in patients with advanced congestive heart failure secondary to ischemic or nonischemic cardiomyopathy. Am J Cardiol 2001; 88: 987-93.

8. Cahalin L, Pappagianopoulos P, Prevost S, Wain J, Ginns L. The relationship of the 6-min walk test to maximal oxygen consumption in transplant candidates with endstage lung disease. Chest 1995; 108: 452-9.

9. Liesker JLW, Wijkstra PJ, Ten Hacken NHT, Köeter GH, Postma DS, Kerstjens HAM. Systematic review of the effects of bronchodilators on exercise capacity in patients with COPD. Chest 2002; 121: 597-608.

10. Diaz O, Begin P, Andresen M, Prieto ME, Castillo C, Jorquera J, et al. Physiological and clinical effects of diurnal noninvasive ventilation in hypercapnic COPD. Eur Respir J 2005; 26: 1016-23.

11. Pinto-Plata VM, Cote C, Cabral H, Taylor J, Celli BR. The 6-min walk distance: change over time and value as a predictor of survival in severe COPD. Eur Respir J 2004; 23: 28-33.

12. Miyamoto S, Nagaya S, Satoh T, Kyotani S, Sakamaki F, Fujita $M$, et al. Clinical correlates and prognostic significance of six-minute walk test in patients with primary pulmonary hypertension. Comparison with cardiopulmonary exercise testing. Am J Respir Crit Care Med 2000; 161: 487-92.

13. Casanova C, Cote CG, Marin JM, de Torres JP, Aguirre- 
Jaime A, Mendez R, et al. The six-minute walk distance: Long term follow up in patients with COPD. Eur Respir J 2007; 29: 535-40.

14. Pérez G, Pinto C, Díaz O, Lisboa C. Deterioro clínico y funcional en pacientes con enfermedad pulmonar obstructiva crónica que han dejado de fumar. Rev Chil Enf Respir 2006; 22: 267.

15. ATS Statement. Guidelines for the six-minute walk test. Am J Respir Crit Care Med 2002; 166: 111-7.

16. Redelmeier DA, Bayoumi AM, Goldstein RS, Guyatt GH. Interpreting small differences in functional status: the six minute walk test in chronic lung disease patients. Am J Respir Crit Care Med 1997; 155: 1278-82.

17. Enright P, Sherrill D. Reference equations for the sixminute walk in healthy adults. Am J Respir Crit Care Med 1998; 158:1384-7.

18. Troosters T, Gosselink R, Decramer M. Six minute walking distance in healthy elderly subjects. Eur Respir J 1999; 14: 270-4.

19. Gibbons W, Fruchter N, Sloan S, Levy R. Reference values for a multiple repetition 6-minute walk test in healthy adults older than 20 years. J Cardiopulm Rehabil 2001; 21: 87-93.

20. Camarri B, Eastwood PR, Cecins NM, Thompson PJ, Jenkins S. Six minute walk distance in healthy subjects aged 55-75 years. Respir Med 2006; 100: 658-65.
21. Chetta A, Zanini A, Pisic G, Aiello M, Tzani P, Neri M, Olivieri D. Reference values for the 6-min walk test in healthy subjects 20-50 years old. Respir Med 2006; 100: 1573-8.

22. Charlson M, Szatrowski TP, Peterson J, Gold J. Validation of a comorbidity index. J Clin Epidemiol 1994; 42: 124551.

23. Miller MR, Hankinson J, Brusasco V, Burgos F, Casaburi R, Coates A, et al. Standardisation of spirometry. Eur Respir J 2005; 26: 319-23.

24. Borg GAV. Psychophysical basis of perceived exertion. Med Sci Sports Exerc 1982; 14: 377-81.

25. Celli BR,Cote CG, Marín JM, Casanova C, Montes de Oca M, Mendez R, et al. The body-mass index, airflow obstruction, dyspnea and exercise capacity index in chronic obstructive pulmonary disease. N Engl J Med 2004; 350: 1005-12.

26. Casanova C, López MV, Marin JM, De Torres JP, Casas A, Montes de Oca M. Six minute walk distance in a multicenter study of healthy subjects aged 40-80 years in Spain and South America. Am J Respir Crit Care Med 2007; 175: A955.

27. Jones NL. Clinical Exercise Testing. $4^{\text {th }}$ ed. Philadelphia: WB Saunders Company; 1997; pp: 243-2.

28. Dempsey JA, Wagner PD. Exercise-induced arterial hypoxemia. J Appl Physiol 1999; 87: 1997-2006. 